\title{
Evaluación interactiva del aprendizaje de Investigación Operativa basada en juegos mediante la plataforma Kahoot!
}

\section{Marina Segura, Concepción Maroto, Concepción Ginestar, José Ramón Navarro, Isabel Martón}

Departamento de Estadística e Investigación Operativa Aplicadas y Calidad. Universitat Politècnica de València. E-mail: masema@upvnet.upv.es; cmaroto@eio.upv.es; cginesta@upvnet.upv.es; jonacer@upvnet.upv.es; ismarllu@upv.es

\begin{abstract}
Emerging technologies and mobile platforms allow the introduction of new teaching and learning strategies, in particular new ways to implement games in higher education. Kahoot! is a free platform, which can be used by teachers to develop games for classroom activities. Although this tool is the most used mobile app and one of the first to be used in education, there are no studies about its application in learning Operations Research. The objective of this paper is to evaluate Kahoot's capabilities for continuous assessment, as well as its contribution in improving students' motivation, learning and performance in decision making tecniques. As positive outcomes we can highlight that the majority of students think Kahoot is a fun experience and that it is also useful when revising concepts. Moreover, Kahoot games increase student'engagement and provide immediate feedback for both teacher and student. In addition, results about student's performance and motivation are presented.
\end{abstract}

Keywords: gamification, Kahoot!, Operations Research, learning, assessment, motivation

\section{Resumen}

Las nuevas tecnologías y aplicaciones móviles facilitan la implantación de nuevas estrategias de enseñanza-aprendizaje y en particular nuevas formas de implementar juegos en educación superior. Kahoot! es una plataforma gratuita con la que el profesor puede crear juegos para utilizar en el aula. Aunque esta herramienta es la aplicación móvil más utilizada y una de las primeras en educación, no hay estudios publicados sobre su utilización en el apredizaje de Investigación Operativa. El objetivo de este trabajo es la evaluación de las prestaciones de Kahoot para la evaluación continua, así como la contribución de esta herramienta para mejorar la motivación, el aprendizaje y el rendimiento de los alumnos en las técnicas de toma de decisiones empresariales. Como resultados positivos podemos destacar que la mayoría de los alumnos consideran que es divertido, que representa una buena herramienta para repasar conceptos. Además aumenta la participación durante las clases y aporta un feedback inmediato tanto al alumno como al 
profesor. Asimismo, presentamos resultados sobre el rendimiento y la motivación de los alumnos.

Palabras clave: gamificación, Kahoot!, Investigación Operativa, aprendizaje, evaluación, motivación

\section{Introducción}

El juego es una herramienta poderosa para el aprendizaje, que combina curiosidad y placer (Mora, 2018). En la última década se ha observado una tendencia creciente en la utilización de juegos en educación, siendo frecuentes en la literatura sobre el tema los términos aprendizaje basado en juegos y gamificación. El concepto gamificación se utiliza desde 2008 y hace referencia a la utilización de elementos característicos de los juegos (puntos, medallas, clasificación) en contextos no lúdicos. En la mayoría de las publicaciones sobre gamificación y aprendizaje basado en juegos estos conceptos están relacionados con los objetivos de mejorar la motivación e involucrar a los participantes (Bozkurt \& Durak, 2018).

Los avances en las tecnologías de la información y comunicación y las aplicaciones móviles nos ofrecen la posibilidad de implantar nuevas estrategias de enseñanza-aprendizaje y en particular nuevas formas de implementar juegos (Plump y Larosa, 2017). En los últimos años se han desarrollado aplicaciones para móviles y web, tales como Kahoot!, Quizizz o Socrative que los profesores pueden utilizar para introducir juegos en educación. Kahoot! es una herramienta gratuita con la que el profesor puede crear cuestionarios para utilizar en el aula con la finalidad de reforzar el aprendizaje y la motivación (Dellos, 2015). Los alumnos se incorporan al juego a través de una App en su móvil, Tablet o computador introduciendo el PIN del cuestionario creado por el profesor. La interfaz es muy atractiva, los alumnos ven las preguntas en la pantalla del aula, introducen su nombre y contestan todos a la vez. Las preguntas están limitadas a 80 caracteres y las respuestas a 60 . El profesor puede modificar el tiempo de respuesta (desde 5 segundos a 2 minutos), las preguntas y añadir imágenes, fotos o vídeos. Finalmente, se muestra el pódium con los tres alumnos que han obtenido mayor puntuación, que depende también del tiempo que el alumno tarda en responder.

Quizizz es también una plataforma gratuita que permite la creación de cuestionarios online de evaluación. La elaboración de los cuestionarios, el formato del juego y la página web son muy similares a los de Kahoot!. En Quizizz las preguntas y las posibles respuestas se muestran individualmente en los dispositivos de los estudiantes. Los tiempos de respuesta pueden ser más largos, desde 5 segundos a 15 minutos. Sin embargo, Kahoot! ocupa el primer puesto en este tipo de aplicaciones y el sexto lugar en el ranking de herramientas para educación, mientras que Socrative ocupa el puesto 56 y Quizizz no aparece en Hart (2018).

Alcover et al. (2018) describen varias experiencias de aplicación de Kahoot! en la enseñanzaaprendizaje de Estadística en la Universitat Politècnica de València (UPV) con la finalidad de captar la atención y motivar al alumno, reforzar el aprendizaje, evaluar competencias transversales y contenidos. Otros casos de estudio han utilizado Kahoot como herramienta de autoevaluación en la universidad (Sempere, 2018).

El empleo de juegos para mejorar el proceso de enseñanza-aprendizaje de Investigación Operativa no es nuevo (Griffin, 2007). Podemos encontrar ejemplos interesantes en Cochran 
(2012, 2015). Recientemente Dias (2017) ha descrito un caso de aplicación de estrategias de gamificación en cursos de Investigación Operativa en Administración de Empresas. Sin embargo, no hemos encontrado en la literatura publicaciones que describan y evalúen la incorporación de aplicaciones móviles como Kahoot! para mejorar la motivación y los resultados en el aprendizaje de Investigación Operativa en los estudios de Administración de Empresas, con la excepción del caso descrito en Guzmán et al. (2018).

\section{Objetivos}

El objetivo general del presente trabajo es la evaluación de las prestaciones y adecuación de las tecnologías y aplicaciones móviles para ampliar el sistema de evaluación continua de Investigación Operativa en el Grado de Administración y Dirección de Empresas, así como la contribución de estas herramientas para mejorar la motivación, el aprendizaje y el rendimiento de los alumnos en las técnicas de toma de decisiones empresariales. Este trabajo se enmarca en un Proyecto de Innovación y Mejora Educativa de la Universidat Politècnica de València (cursos 2018-19 y 2019-20).

Los objetivos específicos son:

1. Analizar los resultados de los cuestionarios realizados con la plataforma Kahoot! que los alumnos responden con el móvil u otro dispositivo en el aula y en el laboratorio durante el curso 2018-19.

2. Analizar la percepción de los alumnos sobre esta experiencia de innovación educativa con la finalidad de mejorar la motivación, incentivar el estudio regular y la competitividad, asi como su aplicación como herramienta de evaluación continua.

En los siguientes apartados se presenta el contexto y el material docente digital desarrollado en la innovación, la metodología y los principales resultados. Por último, se incluyen las conclusiones del estudio y futuras propuestas de mejora educativa.

\section{Desarrollo de la innovación}

\subsection{Contexto}

La Investigación Operativa (IO) es una tecnología de ayuda a la toma de decisiones empresariales que se imparte en $3^{\circ}$ curso del Grado en Administración y Dirección de Empresas (GADE) de la Facultad de Administración y Dirección de Empresas (FADE). Asimismo, esta asignatura se imparte en $4^{\circ}$ curso de dos Dobles Titulaciones de Grado en Ingeniería de Tecnologías y Servicios de Telecomunicación (GITST) y Grado en Ingeniería Informática (GII) con GADE.

La Investigación Operativa es una materia de naturaleza cuantitativa, cuyos resultados de aprendizaje son que los alumnos sean capaces de formular modelos de optimización para resolver problemas reales de toma de decisiones, aplicar los conceptos y algoritmos para resolver modelos de programación lineal, entera, no lineal, multiobjetivo y multicriterio mediante software profesional e interpretar los resultados para mejorar las decisiones empresariales. 
IO tiene 6 créditos ECTS, 3 créditos de teoría y 3 créditos de prácticas, 1 de prácticas de aula y 2 de laboratorio informático. En el aula se combina la presentación de modelos, métodos y aplicaciones por parte del profesor con la realización de casos prácticos por parte de los alumnos, aplicando el aprendizaje colaborativo. En las diez sesiones de dos horas en el laboratorio los alumnos trabajan en grupos formulando modelos, que después resuelven e interpretan las soluciones, con la ayuda del profesor. La implantación de esta metodología, enfocada hacia el aprendizaje de los alumnos, tiene una larga trayectoria y su origen en varios Planes de Innovación Educativa.

El material docente básico está formado por un libro de texto expresamente diseñado para impartir la asignatura, presentaciones del profesor y videos (screencasts) con casos prácticos. Estos últimos están disponibles en abierto como obra digital en el repositorio institucional de la UPV RiuNet, en la colección Polimedia ADE. El libro incluye tanto el material para las explicaciones teóricas como los casos prácticos a desarrollar y resolver en el aula y en el laboratorio informático. Este libro está publicado en castellano e inglés para el grupo ARA y ambos en formato papel y electrónico (Maroto et al., 2012, Maroto et al., 2014). Las presentaciones de las unidades didácticas en PowerPoint, están disponibles en poliformaT para los alumnos, también en castellano e inglés.

El sistema de evaluación actual se basa en la evaluación continua de los informes que los alumnos realizan en las sesiones de prácticas informáticas (20\%) y 2 exámenes (40\% cada uno). Los alumnos que no superan la asignatura pueden realizar un examen de recuperación. La media de la tasa de rendimiento de los estudiantes en los 6 últimos cursos es del 90\%. El porcentaje medio de suspensos es de 7,8\% y el de no presentados $2,2 \%$.

\subsection{Material docente digital}

Durante el curso 2019-20 se han elaborado 9 cuestionarios con la plataforma Kahoot! para utilizar en las clases de teoría y prácticas de aula (5) como en las prácticas de laboratorio (4), todos en castellano e inglés. Tres de los cuatro kahoots de las prácticas de laboratorio se han elaborado para nuevos casos prácticos diseñados en este curso. Todos se han respondido por los alumnos de los 4 grupos que cursan la asignatura en el $1^{\circ}$ semestre y se están utilizando en el $2^{\circ}$ semestre en el grupo de doble titulación GII-ADE.

En la figura 1 se presenta un ejemplo Kahoot diseñado para una clase de teoría de aula con el objetivo de reforzar los principales conceptos de formulación y resolución de modelos de programación lineal. Asimismo, se pretende que los alumnos estudien a lo largo del curso. La figura 2 presenta cómo visualizan los alumnos las preguntas en la pantalla del aula y lo que ven en su dispositivo móvil. A medida que los alumnos introducen el PIN y se conectan al juego aparece en pantalla el número de jugadores.

\subsection{Metodología}

Se ha realizado una encuesta anónima a los alumnos al final del semestre que incluye la valoración de juegos Kahoot en la escala de Likert de 1 a 5, junto con otra información relevante como el grupo, nota del primer parcial, asistencia a clase y meta personal para la calificación de la asignatura. La encuesta completa se presenta en el Anexo. Tras realizar un análisis descriptivo de los resultados de los Kahoots y de la encuesta, se ha analizado si la 
percepción de los alumnos respecto a esta experiencia de innovación está relacionada con otras variables. Este análisis se ha realizado mediante tablas de contingencia, utilizando el test estadístico Chi-cuadrado de Pearson.

\begin{tabular}{|l|l|}
\hline El análisis de sensibilidad analiza el efecto de cambios en \\
\hline Las variables & $\begin{array}{l}\text { Coeficientes de la función objetivo y } \\
\text { coeficientes técnicos }\end{array}$ \\
\hline Coeficientes de la función objetivo y bi (RHS) & Coeficientes técnicos y bi (RHS) \\
\hline El coste de oportunidad es la mejora del valor de la función objetivo por \\
\hline Unidad adicional de recurso & Tener una unidad menos de recurso \\
\hline Depende del problema & Depende del objetivo \\
\hline ¿Qué significa optimizar un objetivo? & Minimizar \\
\hline Maximizar & Todas son correctas \\
\hline Depende del objetivo & \\
\hline ¿Qué evalúa el análisis de sensibilidad? & Efecto de cambios en los coeficientes técnicos \\
\hline La respuesta de la solución óptima & Todas son correctas \\
\hline Rango de las variables & La función objetivo \\
\hline Los coeficientes técnicos en Programación Lineal están en \\
\hline Primer miembro de las restricciones & Todas son correctas \\
\hline Segundo miembro de las restricciones & Negativo \\
\hline Si la variable de holgura de una restricción es positiva su coste de oportunidad es \\
\hline Positivo & No se sabe \\
\hline Cero &
\end{tabular}

Fig. 1 Ejemplo Kahoot! Unidad didáctica: Formulación y Resolución de Modelos de Programación Lineal

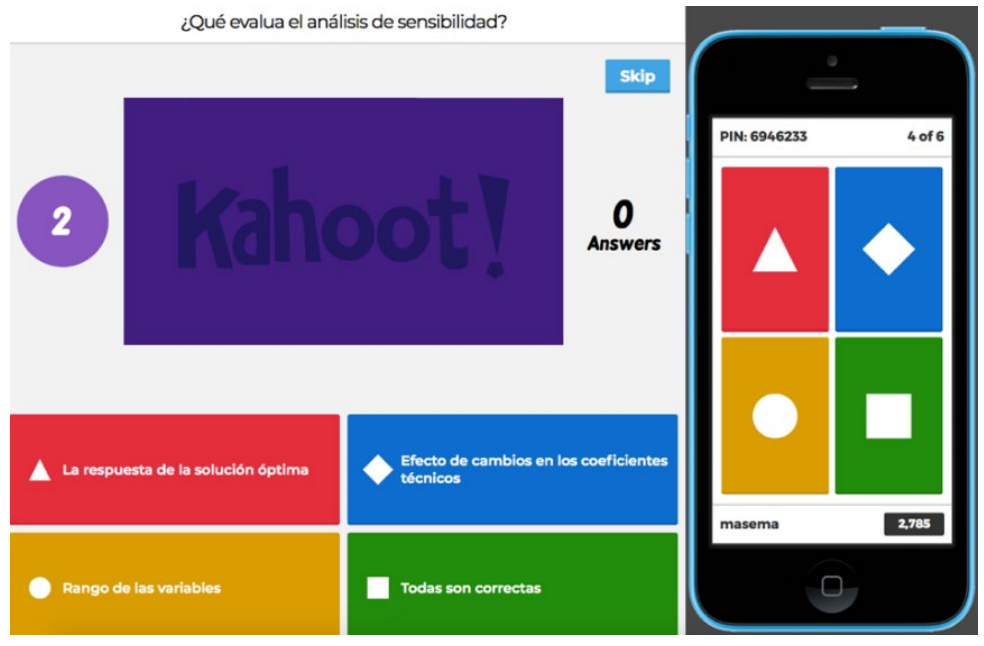

Fig. 2 Ejemplo de visualización en pantalla y en el móvil del alumno de una pregunta en Kahoot!

\section{Resultados}

En el primer semestre del curso 2018/19 se han realizado nueve kahoots en los grupos de GADE y en la doble titulación con telecomunicaciones -GITST-, cuatro de ellos en las sesiones de prácticas de laboratorio y cinco durante las clases de teoría y práctica de aula. No se presentan datos del doble grado con informática porque se imparte en el segundo semestre. 
La tabla 1 recoge el número de alumnos matriculados en cada grupo y el porcentaje de los que han respondido la encuesta, que a nivel global es del $58,01 \%$. Se puede afirmar que tenemos una elevada representatividad tanto a nivel global como en todos los grupos. Los grupos de Tarde y doble grado de ADE-Teleco tienen una menor asistencia a las clases de aula y mayor porcentaje de alumnos con exención de asistencia.

Tabla 1. Número de alumnos matriculados y porcentaje de respuesta por grupos

\begin{tabular}{|l|c|c|c|}
\hline \multicolumn{1}{|c|}{ Grupo } & Matriculados & Respuesta & \% Respuesta \\
\hline Mañanas & 60 & 42 & $70,00 \%$ \\
\hline ARA & 43 & 28 & $65,12 \%$ \\
\hline Tardes & 55 & 24 & $43,64 \%$ \\
\hline Doble ADE-Teleco & 23 & 11 & $47,83 \%$ \\
\hline TOTAL & 181 & 105 & $58,01 \%$ \\
\hline
\end{tabular}

Los resultados de los juegos kahoots se obtienen a partir del número de repuestas correctas y el tiempo que el alumno tarda en contestar la pregunta. Cuanto mayor es el tiempo utilizado en contestar correctamente menor es la puntuación. En el último kahoot de laboratorio y los dos últimos en clases de aula se utilizó un sistema de puntuación extra que otorga más puntos cuando los alumnos encadenan dos respuestas correctas. En las tablas 2 y 3 se recoge la participación y los resultados de los kahoots realizados durante las sesiones prácticas y durante las clases de aula de los cuatro grupos de la asignatura.

Tabla 2. Resultados de los juegos kahoot realizados en las sesiones de prácticas

\begin{tabular}{|c|c|c|c|c|c|}
\hline & & Kahoot P1 ${ }^{1}$ & Kahoot P2 ${ }^{1}$ & Kahoot P3 & Kahoot P10 \\
\hline & $\mathrm{N}^{\circ}$ preguntas & 5 & 6 & 5 & 8 \\
\hline \multirow{4}{*}{$\begin{array}{l}\text { Doble ADE- } \\
\text { Teleco }\end{array}$} & $\mathrm{N}^{\circ}$ Jugadores & 17 & 15 & 16 & 11 \\
\hline & \% Respuestas correctas & $75,29 \%$ & $57,78 \%$ & $83,75 \%$ & $69,32 \%$ \\
\hline & Promedio puntuación & 6,10 & 4,87 & 6,99 & 5,99 \\
\hline & $\%$ de Aprobados & $70,59 \%$ & $46,67 \%$ & $87,50 \%$ & $81,82 \%$ \\
\hline \multirow[t]{4}{*}{ Mañanas } & $\mathrm{N}^{\circ}$ Jugadores & 59 & 23 & 62 & 59 \\
\hline & $\%$ Respuestas correctas & $62,13 \%$ & $55,47 \%$ & $58,70 \%$ & $65,40 \%$ \\
\hline & Promedio puntuación & 4,54 & 4,75 & 4,56 & 5,37 \\
\hline & $\%$ de Aprobados & $31,12 \%$ & $30,43 \%$ & $66,83 \%$ & $83,80 \%$ \\
\hline \multirow[t]{4}{*}{ ARA } & $\mathrm{N}^{\circ}$ Jugadores & & 47 & 55 & 46 \\
\hline & $\%$ Respuestas correctas & & $54,96 \%$ & $48,00 \%$ & $28,26 \%$ \\
\hline & Promedio puntuación & & 4,33 & 4,93 & 3,97 \\
\hline & $\%$ de Aprobados & & $37,89 \%$ & $22,64 \%$ & $24,33 \%$ \\
\hline \multirow[t]{4}{*}{ Tardes } & $\mathrm{N}^{\circ}$ Jugadores & 39 & & 39 & 40 \\
\hline & \% Respuestas correctas & $66,15 \%$ & & $58,46 \%$ & $58,75 \%$ \\
\hline & Promedio puntuación & 5,20 & & 4,64 & 5,46 \\
\hline & $\%$ de Aprobados & $63,95 \%$ & & $49,18 \%$ & $52,26 \%^{2}$ \\
\hline \multicolumn{2}{|c|}{ \% Total de Aprobados } & $55,22 \%$ & $38,33 \%$ & $56,54 \%$ & $60,55 \%$ \\
\hline
\end{tabular}

${ }^{l}$ No se pudo realizar el kahoot en algunos grupos. 
En los kahoots de las prácticas de laboratorio se preguntan cuestiones sobre conceptos necesarios para realizar la práctica de forma adecuada. Además, dos de estos kahoots (prácticas 1 y 3) incluyen preguntas sobre la interpretación del caso práctico resuelto, por tanto, se realizan al final de la sesión. Como los kahoots 2 y 10 solo incluyen conceptos previos se realizaron al principio de la clase.

El porcentaje de alumnos que aprueban los kahoots en los casos prácticos de laboratorio varía entre el $23 \%$ y $84 \%$ dependiendo de la práctica y el grupo. El grupo de ADE-Teleco obtuvo los mejores resultados en las clases prácticas. Además, tiene el mayor porcentaje de aprobados en todos los kahoots, excepto en la práctica número 10. El grupo de mañanas también tiene buenos resultados. Los peores resultados se presentan en el grupo ARA en cuanto a porcentaje de aprobados.

Tabla 3. Resultados del kahoot realizados en las clases de aula

\begin{tabular}{|c|c|c|c|c|c|c|}
\hline & & $\begin{array}{l}\text { Kahoot } \\
\text { Tema } 1 \\
\end{array}$ & $\begin{array}{l}\text { Kahoot } \\
\text { Tema } 2 \\
\end{array}$ & $\begin{array}{l}\text { Kahoot } \\
\text { Tema } 3\end{array}$ & $\begin{array}{c}\text { Kahoot T7 } \\
\text { AHP }\end{array}$ & $\begin{array}{c}\text { Kahoot T7 } \\
\text { PROMETHEE }\end{array}$ \\
\hline & $\mathrm{N}^{\mathrm{o}}$ preguntas & 6 & 6 & 6 & 10 & 9 \\
\hline \multirow{4}{*}{$\begin{array}{c}\text { ADE- } \\
\text { TELECO }\end{array}$} & $\mathrm{N}^{\mathrm{o}}$ Jugadores & 13 & 13 & 11 & 11 & 10 \\
\hline & $\begin{array}{l}\text { \% Respuestas } \\
\text { correctas }\end{array}$ & $58,97 \%$ & $55,13 \%$ & $62,12 \%$ & $58,18 \%$ & $65,56 \%$ \\
\hline & $\begin{array}{l}\text { Promedio } \\
\text { puntuación }\end{array}$ & 4,89 & 4,29 & 5,69 & 5,57 & 6,21 \\
\hline & $\%$ de Aprobados & $69,23 \%$ & $46,15 \%$ & $90,00 \%$ & $63,64 \%$ & $80,00 \%$ \\
\hline \multirow[t]{4}{*}{ Mañanas } & $\mathrm{N}^{\circ}$ Jugadores & 51 & 54 & 50 & 42 & 41 \\
\hline & $\begin{array}{l}\% \text { Respuestas } \\
\text { correctas }\end{array}$ & $53,92 \%$ & $48,77 \%$ & $66,00 \%$ & $52,14 \%$ & $64,50 \%$ \\
\hline & $\begin{array}{l}\text { Promedio } \\
\text { puntuación }\end{array}$ & 4,02 & 3,69 & 4,87 & 4,54 & 5,98 \\
\hline & $\%$ de Aprobados & $27,45 \%$ & $14,81 \%$ & $48,00 \%$ & $38,10 \%$ & $68,29 \%$ \\
\hline \multirow[t]{4}{*}{ ARA } & $\mathrm{N}^{\mathrm{o}}$ Jugadores & 43 & 31 & 35 & 26 & 24 \\
\hline & $\begin{array}{l}\text { \% Respuestas } \\
\text { correctas }\end{array}$ & $50,39 \%$ & $38,17 \%$ & $59,05 \%$ & $54,62 \%$ & $73,15 \%$ \\
\hline & $\begin{array}{l}\text { Promedio } \\
\text { puntuación }\end{array}$ & 3,89 & 2,77 & 4,58 & 4,77 & 6,83 \\
\hline & $\%$ de Aprobados & $37,21 \%$ & $3,23 \%$ & $48,57 \%$ & $34,62 \%$ & $83,33 \%$ \\
\hline \multirow[t]{4}{*}{ Tardes } & $\mathrm{N}^{\mathrm{o}}$ Jugadores & 35 & 24 & 25 & 22 & 22 \\
\hline & $\begin{array}{l}\% \text { Respuestas } \\
\text { correctas }\end{array}$ & $63,81 \%$ & $49,31 \%$ & $65,33 \%$ & $57,73 \%$ & $59,09 \%$ \\
\hline & $\begin{array}{l}\text { Promedio } \\
\text { puntuación }\end{array}$ & 4,90 & 4,05 & 5,86 & 5,69 & 5,31 \\
\hline & $\%$ de Aprobados & $65,71 \%$ & $62,50 \%$ & $96,00 \%$ & $68,18 \%$ & $59,09 \%$ \\
\hline \multicolumn{2}{|c|}{$\%$ Total de Aprobados } & $49,90 \%$ & $31,67 \%$ & $70,64 \%$ & $51,13 \%$ & $72,68 \%$ \\
\hline
\end{tabular}

El grupo de ADE-Teleco de manera general también obtuvo los mejores resultados en los kahoots realizados en las clases de aula. Destaca también positivamente los resultados del grupo de Tardes. En la tabla 3 se observa la reducción del número de alumnos en todos los grupos en los dos últimos kahoots, que corresponden a una unidad didáctica del segundo examen parcial. 
A diferencia de los kahoots realizados en el laboratorio cuya puntuación obtenida corresponde a una parte de la evaluación, los kahoots de las clases de aula no tenían incidencia directa en la nota, sino que otorgaba una puntuación extra a los alumnos que los aprobaba. Esto podría explicar los bajos resultados en algunos de ellos. El análisis de las puntuaciones promedio de los alumnos muestra una concentración en la calificación de aprobado.

La figura 3 muestra la percepción de los alumnos sobre la innovación educativa que representan los juegos kahoots a partir de la encuesta realizada a los alumnos en el curso 2018/19. Los alumnos consideran en su mayoría que los kahoots son bastante o muy divertidos $(65,38 \%)$ y prácticamente la mitad considera que es útil para repasar conceptos (47,12\%). Los grupos que más disfrutan con los kahoots son el grupo de Tardes y el ARA con un $82,6 \%$ y $78,6 \%$ respectivamente. El grupo ARA es el grupo que más importancia le otorga a los kahoots como herramienta útil para repasar conceptos $(71,4 \%)$, siendo esta diferencia significativa con el resto de grupos.

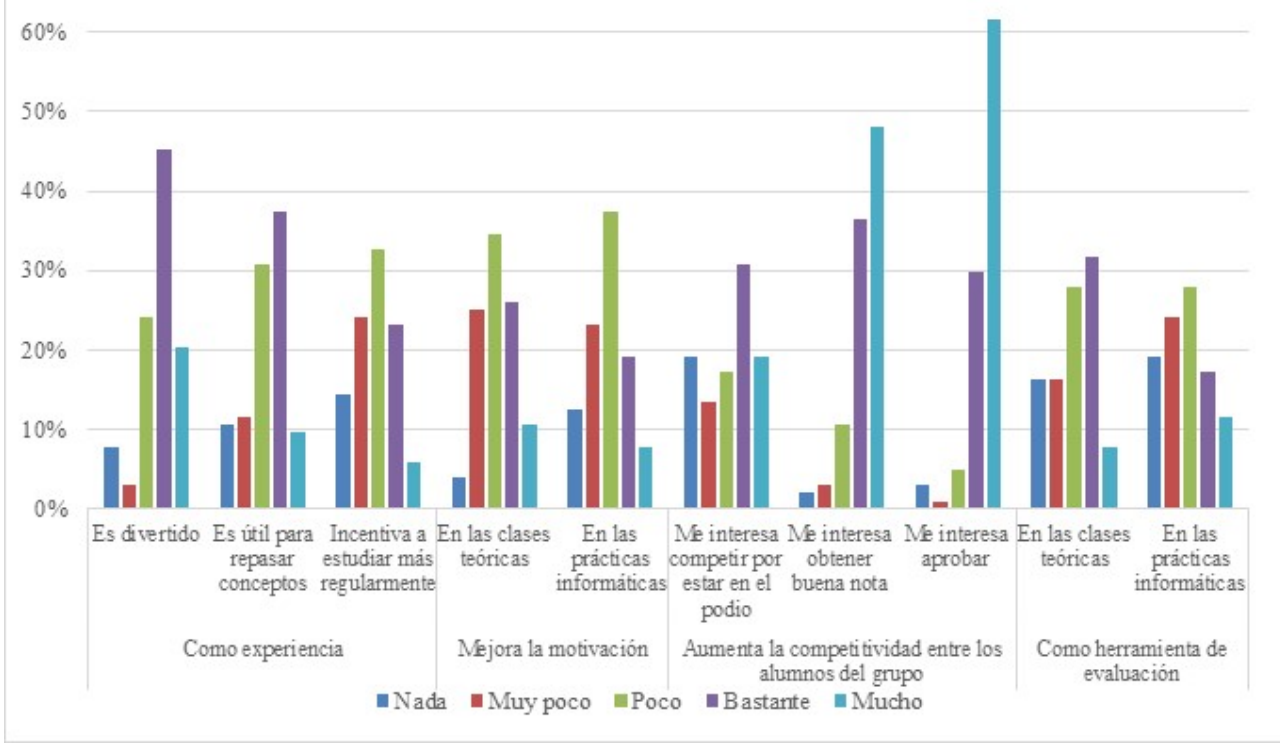

Fig. 3. Resultados de la encuesta de opinión de los alumnos sobre los kahoots realizados en la asignatura Investigación Operativa

El grupo del doble grado ADE-Teleco se diferencia del resto de grupos en la competitividad de los kahoots, dándole menos importancia a las preguntas de "me interesa competir por estar en el podio" " "me interesa obtener buena nota" con un 36,4\% y un 54,5\%. Estos porcentajes en el resto de grupos son, en el grupo ARA 53,6\% y 85,7\%, en el de Mañanas 47,6 y 95,2\% $\mathrm{y}$ en el de Tardes $56,5 \%$ y $78,3 \%$. El comportamiento del grupo de mañanas es significativamente diferente de los demás respecto a que prácticamente todos los alumnos están interesados en obtener buena nota. Este resultado puede estar relacionado con el hecho de que en este grupo se concentran los mejores expedientes, tanto de entrada en la titulación 
como en las notas del grado. En relación con la pregunta de "me interesa aprobar" la mayoría de los alumnos en los cuatro grupos dan una valoración de bastante o mucho. Este dato a nivel global se observa en la figura 3 .

En cuanto a las preguntas sobre la opinión de los alumnos sobre los kahoots como elemento motivador y evaluador de la asignatura de IO, se puede observar que no se da mucha importancia a estos aspectos tanto en las sesiones de prácticas como en las clases de aula, siendo esta opinión compartida por todos los grupos. Como sistema de evaluación únicamente el grupo de tardes le da algo más de importancia y destaca el grupo de ADETeleco donde todos los alumnos lo califican con poca o ninguna preferencia.

Tanto en los comentarios de la encuesta como a lo largo del curso, algunos alumnos consideraban que el tiempo establecido para contestar las preguntas, de 20 o 30 segundos según el nivel de dificultad, era pequeño y tendría que ser mayor.

En cuanto al rendimiento del curso el porcentaje de aprobados ha sido del $57 \%, 20 \%$ de notables, y 7\% sobresalientes. Estos datos son similares al curso previo, con un aumento en el porcentaje de alumnos con sobresalientes y matrículas de honor, $7 \%$ respecto a $5 \%$ del año anterior.

\section{Conclusiones}

La introducción de los juegos basados en la plataforma Kahoot durante curso 2018/19 en Investigación Operativa en GADE, tanto en prácticas de laboratorio como en las clases de aula, pretendía ampliar el sistema de evaluación continua en la asignatura, así como mejorar la motivación, el aprendizaje y el rendimiento de los alumnos.

Comparando con trabajos previos de otros autores que destacan la utilidad de la gamificación para aumentar la motivación no podemos afirmar que los resultados de nuestra experiencia estén en esta línea (Plump y Larosa, 2017; Alcover et al., 2018; Bozkurt y Durak, 2018; Gúzman et al., 2018). Esto se puede deber a que el tiempo que el alumno tarda en contestar una pregunta afecta negativamente a la puntuación, que unido a un tiempo máximo insuficiente, haya actuado como elemento desmotivador. Para paliar este efecto negativo, ya en el propio curso hemos introducido estrategias tales como otorgar puntos extras cuando encadenan varias respuestas correctas (Kahoots AHP y PROMETHEE), aumentar el tiempo de respuesta o considerar la nota máxima como la nota del mejor alumno si ha respondido correctamente todas las preguntas.

Un resultado positivo de nuestra experiencia con Kahoot es que la mayoría de los alumnos consideran que es divertido y representa una buena herramienta para repasar conceptos y aumenta la participación durante las clases. Estos resultados coinciden con los obtenidos en otros trabajos como el Plump y Larosa (2017). También aumenta la competitividad para obtener buenas notas y estar en el podio de los tres mejores. Además, hemos contrastado que es una experiencia muy positiva desde el punto de vista del feedback que proporciona no sólo al alumno sino también al profesor. Por tanto, en el primer caso puede considerarse una forma divertida de autoevaluación y en el segundo ofrece una información instantánea sobre el nivel de conocimientos y comprensión a nivel global. 
Evaluación interactiva del aprendizaje de Investigación Operativa basada en juegos mediante la plataforma Kahoot!

Por último, la principal limitación de estos resultados se debe a que corresponden a un único curso. Por tanto, los autores continuaremos aplicando esta estrategia en los próximos cursos reforzando los resultados positivos y aplicando nuevas estrategias que permitan reducir los negativos.

\section{Referencias}

ALCOVER, R.M., CALDUCH-LOSA, A., VIDAL-PUIG, S. (2018). "Nos divertimos y aprendemos con Kahoot! en las clases de Estadística". En Congreso In-Red 2018. UPV, 19 y 20 de julio. Doi: http://dx.doi.org/10.4995/INRED2018.2018.8642

BOZKURT, A., \& DURAK, G. (2018). "A systematic review of research: In pursuit of homo ludens" en The International Journal of Game-Based Learning, 8 (3), 15-33.

COCHRAN, J.J. (2012). "You want them to remember? Then make it memorable! Means for enhancing operations research education" en European Journal of Operational Research 219, 659-670.

COCHRAN, J.J. (2015). Extending "Lego ${ }^{\circledR}$ My Simplex" en INFORMS Transactions on Education 15(3):224-231. https://doi.org/10.1287/ited.2015.0139.

DELLOS, R. (2015). “Kahoot! A digital game resource for learning” en International Journal of Instructional Technology and Distance Learning. 12 (4), 49-52.

DIAS, J. (2017). "Teaching operations research to undergraduate management students: The role of gamification" en The International Journal of Management Education 15, 98-111

GRIFFIN, P. (2007). "The Use of Classroom Games in Management Science and Operations Research" en INFORMS Transactions on Education 8(1):1-2. https://doi.org/10.1287/ited.8.1.1

GUZMÁN, A., MENDOZA, J., TAVERA, N (2018). "Kahoot!: un mecanismo de innovación para la educación universitaria". El compromiso académico y social a través de la investigación e innovación educativas en la Enseñanza Superior. 663-640. Editorial Octaedro.

HART, J. (2018). Top tools for learning. https://www.toptools4learning.com [6 agosto 2018].

KAHOOT! https://kahoot.com/[Consulta: 10 de septiembre de 2018]

MAROTO, C. et al. (2012). Investigación Operativa en Administración y Dirección de Empresas. Valencia: Editorial UPV. Disponible en papel y libro electrónico.

MAROTO, C. et al. (2014). Operations Research in Business Administration and Management. Valencia: Editorial UPV. Disponible en papel y libro electrónico.

MORA, F. 2018. Neuroeducación. Solo se puede aprender aquello que se ama. Alianza Editorial.

PLUMP, C., LAROSA, J. (2017). "Using Kahoot! in the classroom to create engagement and active learning: a game-based technology solution for elearning novices" en Management Teaching Review, 2(2), 151-158.

QUIZIZZ https://quizizz.com/ [Consulta: 20 de septiembre de 2018]

UNIVERSITAT POLITÈCNICA DE VALÈNCIA (2019). RiuNet. https://riunet.upv.es/

SEMPERE, F. (2018). "Kahoot como herramienta de autoevaluación en la universidad". En Congreso In-Red 2018 UPV, 19 y 20 de julio de 2018 Doi: http://dx.doi.org/10.4995/INRED2018.2018.8730 


\begin{abstract}
ANEXO
Proyecto de Innovación y Mejora Educativa

PIME B30- curso 2018-19

Preferencias sobre el material docente y sistema de evaluación de Investigación Operativa

El objetivo esta encuesta es conocer las preferencias de los alumnos por los diferentes tipos de materiales docentes y sistema de evaluación utilizados en Investigación Operativa.
\end{abstract}

\title{
1. Indica el grupo en el que estás matriculado/a:
}

Grupo Mañanas

Grupo Tardes

Grupo ARA

Grupo Doble ADE- ITS Telecomunicación

Grupo Doble ADE- Ingeniería Informática

2. Grado de utilización del material docente en el aprendizaje de Investigación Operativa.

Escala de 1 (nada) a 5 (mucho).

\begin{tabular}{|l|c|c|c|c|c|}
\hline \multicolumn{1}{|c|}{ Material docente } & $\mathbf{1}$ & $\mathbf{2}$ & $\mathbf{3}$ & $\mathbf{4}$ & $\mathbf{5}$ \\
\hline Biblioteca/Personal & Nada & Muy poco & Poco & Bastante & Mucho \\
\hline $\begin{array}{l}\text { Libro: Investigación Operativa en } \\
\text { ADE }\end{array}$ & & & & & \\
\hline Book: Operations Research in BAM & & & & & \\
\hline En Poliforma T/Recursos: & & & & & \\
\hline Presentaciones de clase & & & & & \\
\hline Videos de Casos Prácticos & & & & & \\
\hline Otros recursos & & & & & \\
\hline $\begin{array}{l}\text { PoliformaT: } \\
\text { resueltos/Answer Key }\end{array}$ & & & & & \\
\hline $\begin{array}{l}\text { PoliformaT: Material } \\
\text { vomplementario/ Supplementary }\end{array}$ & & & & & \\
\hline Otros: indicar & & & & \\
\hline
\end{tabular}


Evaluación interactiva del aprendizaje de Investigación Operativa basada en juegos mediante la plataforma Kahoot!

3. Indica tus preferencias por los distintos tipos de materiales docentes aplicando el método AHP basado en comparaciones por pares y la escala de 1 a 9.

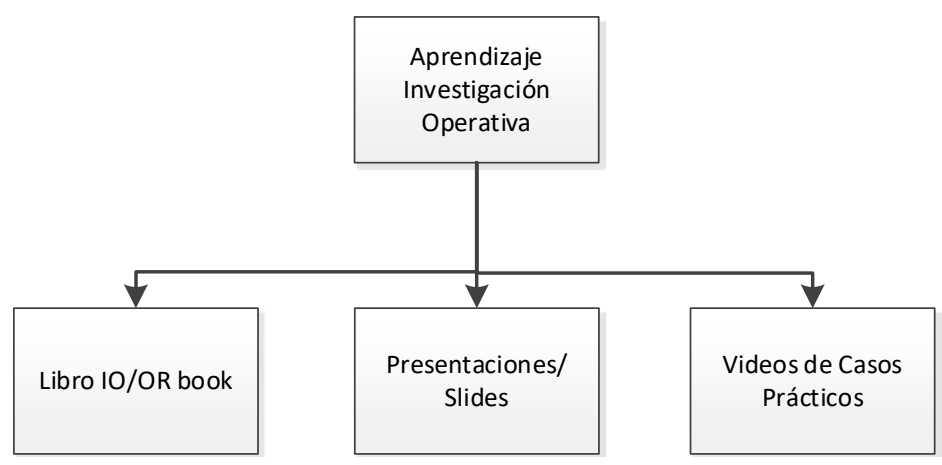

Compara la importancia relativa del material docente indicado en la fila de la matriz de comparaciones con el de la columna utilizando la escala del método AHP.

Escala:

1 igual importancia, 3 importancia moderada, 5 importancia fuerte, 7 mucha mayor importancia y 9 importancia extrema del primer elemento sobre el segundo.

Si el material de la columna tiene para ti mayor importancia que el de la fila: 1/3, 1/5, 1/7 y 1/9.

Rellena sólo las tres casillas en blanco.

\begin{tabular}{|c|c|c|c|}
\hline Aprendizaje IO/OR & $\begin{array}{c}\text { Libro } \\
\text { IO/Book OR }\end{array}$ & Presentaciones/Slides & $\begin{array}{c}\text { Videos Casos } \\
\text { Prácticos }\end{array}$ \\
\hline Libro IO/Book OR & 1 & & \\
\hline Presentaciones/Slides & & 1 & 1 \\
\hline Videos Casos Prácticos & & & \\
\hline
\end{tabular}

Si tienes dudas sobre cómo rellenar esta matriz de comparaciones, contesta a las preguntas siguientes marcando la respuesta que refleje mejor tus preferencias.
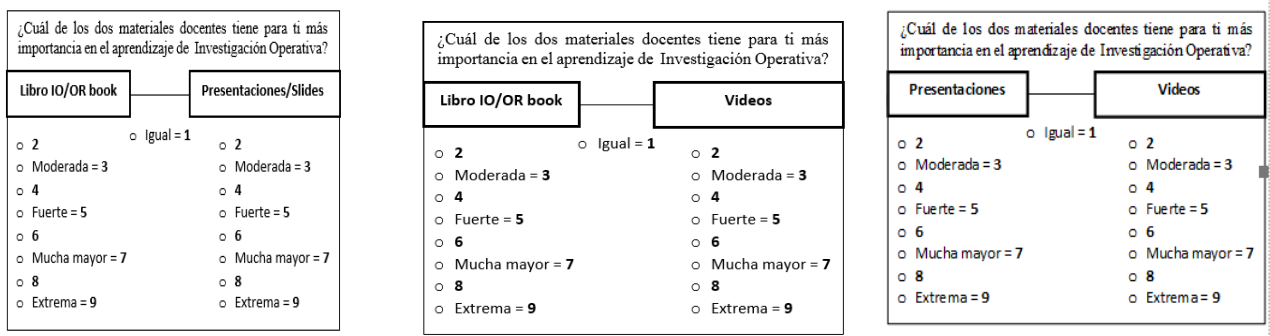

(c)) EY-NC-ND 2019, Universitat Politècnica de València 
4. Experiencia con la utilización de juegos Kahoot en la asignatura Investigación Operativa

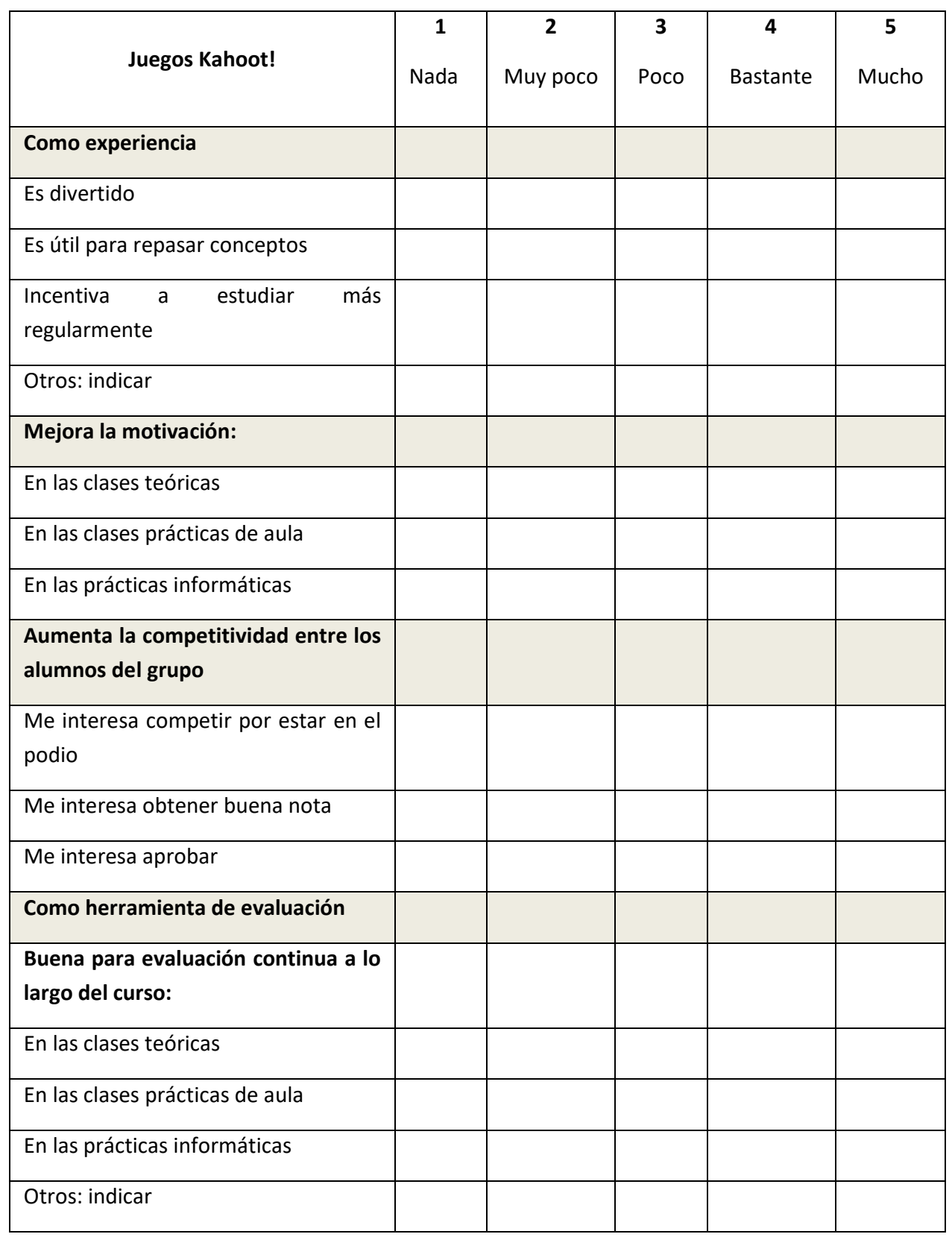

Comentarios y sugerencias personales sobre la utilización de juegos Kahoot en la asignatura Investigación Operativa 
Evaluación interactiva del aprendizaje de Investigación Operativa basada en juegos mediante la plataforma Kahoot!

5. Calificación en la primera parte de la asignatura (Examen + Prácticas laboratorio):
No presentado
Suspenso
Aprobado
Notable
Sobresaliente

6. Asisto regularmente a las clases:

\section{Teóricas y Prácticas de aula}

SI

NO

\section{Prácticas Informáticas}

SI

NO

En caso de NO asistencia indica el motivo:

Prácticas de empresa

Prefiero estudiar con las presentaciones de clase/libro básico asignatura

Otros (indica el motivo):

7. Meta personal para la calificación final de la asignatura Investigación Operativa

Aprobado

Notable

Sobresaliente

Sobresaliente $10 \mathrm{MH}$

8. Sugerencias de mejora para la elaboración de materiales docentes y sistema de evaluación de la asignatura:

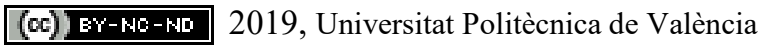

